\title{
Chemical bonding origin of the thermoelectric power factor in Half-Heusler semiconductors \\ Kasper Tolborg
}

Department of Materials, Imperial College London, Exhibition Road, London SW7 2AZ, United Kingdom

k.tolborg@imperial.ac.uk

Thermoelectric materials are able to interconvert thermal and electrical energy, and thus offer the potential to harvest waste heat through solid-state devices. A particularly interesting class of thermoelectric materials are the cubic intermetallic Half-Heusler semiconductors with XYZ stoichiometry, which show promising high temperature thermoelectric properties commonly attributed to the high degeneracy of carrier pockets in the band structure and weak electron-phonon coupling.

Half-Heuslers crystallize with $\mathrm{YZ}$ and $\mathrm{XY}$ forming tetrahedrally coordinated zinc blende networks, and $\mathrm{XZ}$ forming a rock salt network. Stable stoichiometric Half-Heuslers have valence electron counts of 8 or 18, which has led to interpretation of their bonding and properties within Zintl chemistry [1]. Applying Zintl chemistry to Half-Heuslers, the electroposive cation, $\mathrm{X}^{\mathrm{n}+}$, donates all its valence electrons to the covalently bonded [YZ] $]^{\mathrm{n}-}$ polyanion, which then fulfils the 8 - or 18-electron rule. Thus, ionic and covalent bonding patterns coexist and there is a clear distinction between the bonding within the polyanion and the bonding between formal cation and polyanion. Zintl chemistry is often applied for engineering thermoelectric materials, and for Half-Heuslers, it gives rise to predictions regarding the electronic structure and defect chemistry.

Expanding on previous investigations on chemical bonding in Half-Heuslers [2,3], we present the results of computational real space chemical bonding analysis using Bader's quantum theory of atoms in molecules and delocalization indices on a range of Half-Heusler semiconductors. This shows strong deviations from predictions from Zintl chemistry for transition metal based materials and reveal interesting relations between chemical bonding and thermoelectric and response properties [4].

We construct a map of chemical bonding in Half-Heuslers based on real space indicators [5], onto which we map important calculated thermoelectric properties. This reveals that strong covalent bonding between formal cation and polyanion results in increased carrier pocket degeneracies, and therefore improved thermoelectric properties. Thus, the materials least in line with the commonly applied Zintl concept are in fact the ones with the best thermoelectric properties.

This works extends our previous studies showing the inability of Zintl chemistry to explain the unexpected isotropic properties in thermoelectric $\mathrm{Mg}_{3} \mathrm{Sb}_{2}$ [6], and thus presents a critical view on the simplistic chemical concepts too often applied for rational materials design. Furthermore, it highlights the potential of applying tools from chemical bonding analysis and quantum crystallography for materials design.

[1] Zeier, W. G., Schmitt, J., Hautier, G., Aydemir, U., Gibbs, Z. M., Felser, C., Snyder, G. J. (2016). Nat. Rev. Mater. 1, 16032

[2] Bende, D., Grin, Y., Wagner, F. R. (2014). Chem. Eur. J. 20, 9702-9708

[3] Bende, D., Wagner, F. R., Grin., Y. (2015). Inorg. Chem. 54, 3970-3978

[4] Tolborg, K., Iversen, B.B. (2021). Submitted

[5] Raty, J. Y., Schumacher, M., Golub, P., Deringer, V. L., Gatti, C., Wuttig, M. (2019). Adv. Mater. 31, 1806280

[6] Zhang, J., Song, L., Sist, M., Tolborg, K., Iversen, B. B. (2018). Nat. Commun. 9, 4716

Keywords: Chemical bonding; thermoelectric materials; quantum crystallography; materials science 